\title{
Ärztliche Leadership - die nächste Runde
}

\author{
Christof Schmitz ${ }^{\mathrm{a}, \mathrm{d}}$, Matthias Egger ${ }^{\mathrm{b}, \mathrm{e}}$, Peter Berchtold ${ }^{\mathrm{c}, \mathrm{d}, \mathrm{e}}$ \\ ${ }^{a}$ Dr.; ${ }^{b}$ Prof. Dr.; ${ }^{c}$ PD Dr.; ${ }^{\text {d }}$ college M, Bern; ${ }^{e}$ Institut für Sozial- und Präventivmedizin, Universität Bern
}

Spitäler treten in eine neue Epoche ein. Hatten in den letzten Jahren die noch bestehenden Wachstumsmöglichkeiten für common ground betriebswirtschaftlicher und ärztlicher Führung gesorgt, sind nun intensivere Auseinandersetzungen über Wirksamkeit, Wirtschaftlichkeit und Zweckmässigkeit zu erwarten. Kliniken und damit ärztliche Führung geraten unter mehrfachen Druck. Wie kann sich darin ärztliche Leadership weiterentwickeln? Und wie sind die verschiedenen Kaderstufen davon betroffen?

1982 sprach Harvard-Professor John Kotter erstmals von Leadership. Er wies damals darauf hin, dass die Unternehmensumwelten nun rasch wandeln würden und Führung sich darauf einzustellen hätte. Reines Managen - er meinte damit ein Art Verwalten der gegebenen Verhältnisse - würde nicht mehr ausreichen. Proaktiver Wandel sei nun gefragt, mit anderen Worten: Leadership ist erforderlich. Seither ist das Thema Leadership, zuvor gänzlich der politischen Domäne zugehörig, in die Führung von Organisationen eingeschrieben. Was die 80er Jahre für Unternehmen waren, bedeutet die gegenwärtige Zeit für Spitäler und andere Organisationen des Gesundheitssystems: Eine neue Episode beginnt. Sie ist das Resultat einer seit Jahren laufenden Entwicklung, die nun immer unmittelbarer für die beteiligten Akteure spürbar wird. So spürbar, dass professionelle Selbstverständnisse und ärztliche Führung im Kern davon betroffen sind. Weniger denn je reicht das blosse "Organisieren» des klinischen Betriebs, mehr denn je ist Leadership gefragt. Und Leadership meint dabei nicht markante hierarchische Positionsbezüge, sondern die Gestaltung eines medizinischen Angebots in den Spannungsfeldern der konkreten medizinischen, wirtschaftlichen, mitarbeiterbezogenen und gesellschaftlichen Dynamiken.

\section{Symptome des Wandels}

Drei Beispiele sollen symptomatisch zeigen, wie vielfältig der Wandel verläuft und warum nun von einer neuen Episode zu sprechen ist.

- Symptom 1: Letztes Jahr war es so weit. In auffällig vielen Spitälern konnten die projizierten Budgets nicht oder kaum mehr eingehalten werden. Erstmals zeigte sich, dass das Wachstum, auf das man seit Einführung der DRGs - auf betriebswirtschaftlicher wie ärztlicher Seite - erfolgreich gesetzt hatte, nicht mehr eintraf. Ohne exakt angeben zu können, was den Unterschied ausmacht (und das ist das wirklich Beunruhigende), wurde spürbar, dass ein Plafond erreicht ist, aber noch wenig Lösungsideen vorhanden sind, was nun zu tun wäre. Getrieben werden wird diese Entwicklung durch die zunehmende "Ambulantisierung» bislang lukrativer Geschäftsfelder der Spitäler. Beispiele bieten ambulante Operationszentren, die teils kooperativ, teils konkurrenzierend mit den Spitälern von finanzstarken ambulanten Anbietern aufgebaut werden.

- Symptom 2: Die grossen Spitäler, die die Speerspitze der Spezialisierung der Medizin bilden, beginnen sich aktuell neu zu positionieren und zu gruppieren. Exemplarisch zeigt sich das in zwei deutschsprachigen Universitätsspitälern, Bern und Basel, die sich in einer Reorganisation im Rahmen einer umfangreichen Fusion befinden, oder an der Neuordnung der kantonalen stationären Versorgung durch die Spitalverbunde St. Gallen. In allen drei Fällen spielt die Frage eine zentrale Rolle, wie sich eine weiter spezialisierende Medizin unter enger werdenden ökonomischen Parametern und unter Druck von Fallzahlen und Spitallisten erfolgreich aufstellen kann. Die Frage betrifft unmittelbar ärztlich-professionelle Selbstverständnisse: Wie können Netzwerke von Spezialisten sinnvoll und effektiv organisiert werden? Wie kann das Zusammenspiel zwischen Spital, Zuweisenden und nachgelagerten Versorgungsein- 
richtungen bzw. zwischen Zentrum und Peripherie neu gedacht werden?

- Symptom 3: Sowohl Studien wie konkrete Rekrutierungsschwierigkeiten in einigen Disziplinen lassen erkennen, dass Chefarztpositionen an Attraktivität verlieren. Das ist Grund für ernsthafte Sorge. Will der Nachwuchs nicht mehr aufsteigen, ist in Tat und Wahrheit Not am Mann / an der Frau. Dann müssen sich Stellen und Stellenprofile oder, dramatischer, ganze Berufsbilder ändern. Weil die bisherigen Attraktoren wie Autonomie, Status und Einkommen nicht mehr hinreichend wirken, sind neue zu finden. Innovative Konzepte dafür wären vorhanden. Exemplarisch zeigen Beispiele einer distributed oder shared leadership, dass Aufgaben und Verantwortlichkeiten über verschiedene Führungsebenen und -funktionen hinweg verteilt werden können.

Diese drei Symptome - Spezialisierung, verstärkter ökonomischer Druck, Attraktivitätsverlust ärztlicher Leitungspositionen - sind wesentliche Treiber eines Wandels ärztlicher Führung (siehe Abb. 1). Der Treiber «Spezialisierung» betrifft sowohl die Ausgestaltung des Leistungsangebots (welche Patienten, welche Krankheitsbilder, welche Qualität?) als auch Vernetzungsnotwendigkeit mit und zwischen Spezialisten (Stichworte: Interdisziplinarität, Interprofessionalität). Der Treiber «ökonomischer Druck» verschärft die Führungsaufgabe, betriebswirtschaftliche und medizinische Ansprüche zu balancieren. Die Kernfrage hier lautet: Wie kann Leistung gesteigert werden, wenn Wachstum kaum mehr möglich ist? Mit welchen Prozessen und welcher Medizin kann man nachhaltig erfolgreich sein? Der dritte Treiber «Attraktivitätsverlust» bedeutet, dass

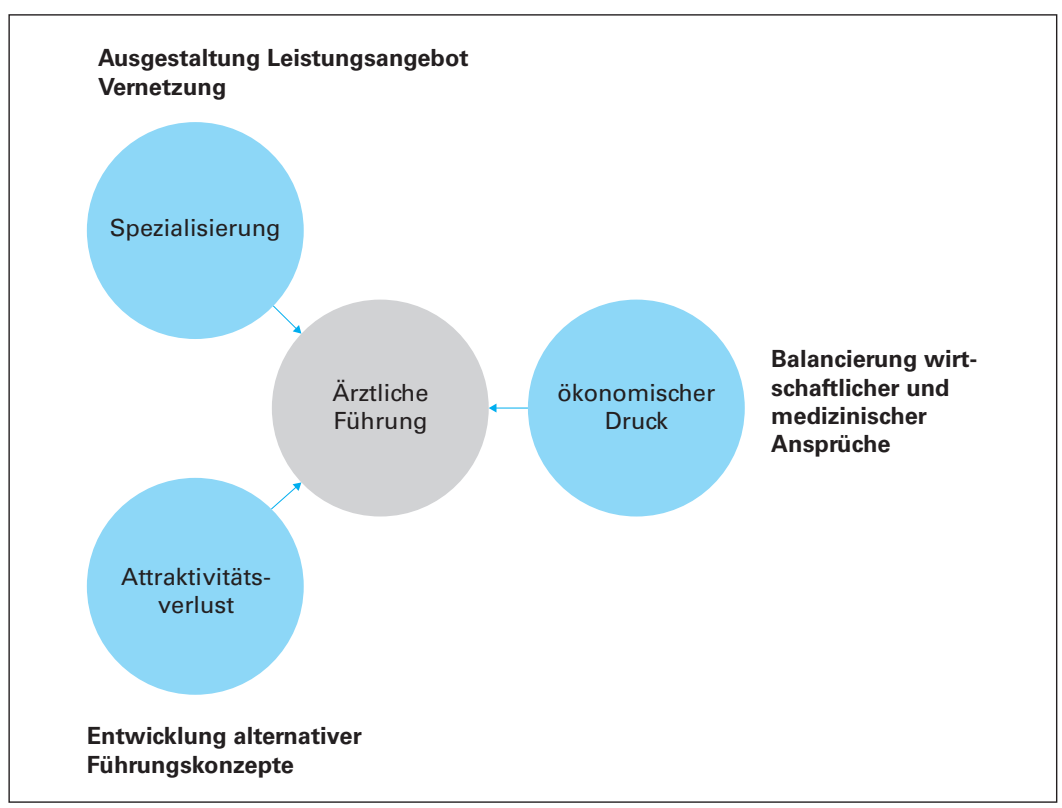

Abbildung 1: Treiber des Wandels ärztlicher Führung. neue, für Nachwuchstalente und die jüngeren Generationen motivierende Führungskonzepte zu finden sind.

\section{Wandel ärztlicher Führung}

Ohne Zweifel ist die ärztliche Führung in den letzten Jahren in Bewegung gekommen. Auch wenn heute weniger hierarchisches Gehabe als in den Dekaden davor die Regel ist, bleibt das Modell der medizinischen wie ökonomischen Letztverantwortung durch den Chefarzt bestehen. Die Chefärztin fungiert als Referenzpunkt sowohl der Sicherheit, der Setzung und der Einhaltung von Leistungs- und Qualitätsstandards als auch des betriebswirtschaftlichen Erfolgs der Klinik. Dieses Fungieren als doppelter Referenzpunkt kann zögern lassen, Verantwortlichkeiten zu teilen. Das ist unproblematisch, solange sich fachliche Kompetenzen sowie ökonomische Entwicklungen überblicken und gut vorhersehen lassen. Die Anforderungen an medizinische wie ökonomische Verantwortlichkeit befinden sich nun aber, wie gezeigt, in dramatischer Veränderung.

Die Frage ist nicht nur, wie Chefärzte und -ärztinnen ihren Weg in dieser neuen Welt finden, sondern ebenso was das für die Kaderärzte insgesamt bedeutet. Was sind die Beiträge und der Stellenwert von Leitenden und Oberärztinnen unter diesen neuen Bedingungen? Sind beispielsweise die Leitenden Ärzte für eine Subspezialität der Klinik zuständig und verantworten sie diese? Sind sie Teil der Klinikleitung? Sind Sie Stellvertreter des Chefs oder fristen sie quasi in einer ökologischen Nische die Existenz eines «Unberührbaren»? Aktuell finden wir in den Kliniken unterschiedliche historisch gewachsene und teilweise stark personengetriebene Formen. Oftmals bleibt die Position der Leitenden Ärzte zwischen Chef- und Oberärzten unscharf. Und wie steht es um die Oberärztinnen? Sie bilden die Basis des klinischen Geschäfts und supervidieren die Assistentinnen und Assistenten. Aber wie weit reicht ihr Handlungsspielraum, wie viel Verantwortung können, wollen und sollen sie übernehmen? Welche Initiativen gehen von ihnen aus?

Ging traditionell chefärztliche Verantwortlichkeit mit grosser Autonomie, hohem Status und gutem Einkommen einher - und war es entsprechend attraktiv, Verantwortung zu akkumulieren -, finden sich mittlerweile Chefärztinnen und -ärzte eng in spital- und medizinübergreifende Führungskonfigurationen eingesponnen. Das verkleinert ihre Kapazitäten, sich um Medizin zu kümmern, verringert ihre Autonomie und verändert die Möglichkeiten zu gestalten. Das setzt nicht nur sie selbst unter Druck, sondern wird auch auf den anderen Kaderebenen beobachtet und löst dort Fragen aus wie «Warum sollte ich mir solches antun?» 
(Originalzitat). Resultat dieser Dynamik ist momentan gelegentlich eine Art Patt zwischen eventuell «abgabewilligen» Chefärzten und irgendwie «unwilligen» anderen Kaderärzten. Eine wenig erfreuliche Situation, die Ausdruck einer allseits ambivalenten Perzeption ist.

\section{Auf der Suche nach Alternativen}

Bilden die aktuellen Modelle die einzige Möglichkeit? Diskutiert finden wir zwei Alternativen. Zum einen die angelsächsische Variante des Consultant-Systems mit einem Chairman, der vor allem koordinative Funktionen wahrnimmt, nicht aber die Sicherheits- und Verantwortlichkeitsfunktion des deutschsprachigen Pendants erfüllen muss. Das ist ein wichtiger struktureller Unterschied. Die einzelnen Leitenden sind dann typischerweise für medizinische Teilgebiete zuständig, Spezialisierung kann so organisiert und Verantwortung auf mehrere Schultern verteilt werden. Das birgt die Chance höherer Kollegialität, kann aber auch eine Perpetuierung der üblichen ärztlichen Führung nun eben auf Abteilungsebene bedeuten.

Literatur

Deerberg-Wittram J, Huland $\mathrm{H}$, Graefen M (Hrsg.). Das MartiniPrinzip. Spitzenmedizin durch Spezialisierung, Ergebnistransparenz und Patientenorientierung. Berlin 2018 Schmitz C, Zwahlen M, Berchtold P. Das Management der Medizin Schweiz Ärzteztg. 2018;99(33): $1078-80$
Eine weitere Alternative stellt das Faculty-System dar, beispielsweise der Martini-Klinik in Hamburg, in dem mehrere «Chefärzte» gleichzeitig und gemeinsam für ein eng umgrenztes Gebiet - im Falle der Martini-Klinik ist es das Prostatakarzinom - verantwortlich zeichnen. Die Besonderheit dieser Variante besteht darin, dass mit hochgradiger Spezialisierung, konsequent auf das Patientenwohl ausgerichteten Strukturen, Abläufen und Kultur sowie der systematischen und transparenten Nutzung von Ergebnisqualitätsmessungen (z.B. PROMS) gemeinsames Lernen und kollektiv getragene Entwicklung möglich werden. Das Martini-Modell findet hier Erwähnung, weil es auf dreierlei Dinge aufmerksam macht. Erstens dass alternative Führungsformen ein verändertes Gesamtsetting einer Klinik (Angebotsspektrum, Organisation) bedingen können; zweitens dass gemeinsames Lernen, basierend auf Qualitätsdaten und Patientenrückmeldungen, neue Führungsformen ermöglichen kann, weil die
Entwicklung von Leistungsstandards und das Monitoring ihrer Einhaltung nicht mehr nur Chefsache sind, sondern eine gemeinsame Aufgabe werden; drittens zeigt das Martini-Modell, dass die Ausrichtung auf eine gute Medizin, die sich konsequent auf patientenzentrierte Feedbacks stützt, wirtschaftlichen Erfolg bringen kann. Auch wenn das Modell nicht beliebig kopierbar ist, die Ingredienzen seines Erfolgs - klare Ausrichtung, datengestütztes Lernen, patientenorientierte Abläufe und Teammodelle - können auch in anderen Klinikentwicklungen hilfreich sein und helfen, einer zusehends problematischeren Polarität von Medizin und Ökonomie zugunsten einer medizingestützten Strategie zu entkommen.

\section{Shared Leadership}

Im Martini-Modell ist nicht mehr ein Chefarzt, eine Chefärztin für Leistung und Qualität letztverantwortlich. Verantwortung wird von einer Faculty getragen. Die Basis dafür liegt in der Messung und Transparenz der Ergebnisqualität und in der gemeinsamen Klarheit über den Umgang damit. Exakt in dieser Gemeinsamkeit liegt ein wesentlicher Unterschied zu traditionellen ärztlichen Führungsformen. Ähnliches lässt sich auch in den aktuellen Entwicklungen in Wirtschaftsorganisationen zu Themen wie "Agile» und «Selbstorganisation" beobachten. Abbau von Hierarchie ist dann sinnvoll und funktioniert, wenn Führungsaufgaben, die klassischerweise in die Vorgesetzten-Mitarbeiter-Beziehung eingebettet sind, lateral - zwischen Peers - wahrgenommen werden können. Exemplarisch: Leistungsbeurteilung kann von der Chefin vorgenommen werden oder zwischen Peers stattfinden. Die Funktionalität von Leistungsbeurteilung (Sind wir entsprechend unseren Erwartungen unterwegs? Wo weichen wir warum ab? Was können wir tun?) ist nicht an Hierarchie gebunden, erfordert im lateralen System aber klare Spielregeln. Schon diese Klarheit entlastet von hierarchischen Abhängigkeitsbezügen. Die entstehende Freiheit hat natürlich die Bereitschaft zu vermehrter Verantwortungsübernahme und Transparenz zur Folge. Führungsaufgaben können über die Klinik «verteilt» werden. Die Folge ist, dass weniger Führungslast auf den Schultern des einen Chefs, der einen Chefin ruht und Leadership stärker auf gemeinschaftliches Engagement aufbaut. So kann neue Attraktivität für «hohe» Positionen entstehen - wenn man sich gemeinsam mit anderen dafür engagiert, hohe medizinische Qualität, die Rendite erzeugt, zu liefern. Da können selbst jüngere Ärztegenerationen $(\mathrm{Y}, \mathrm{Z}$, ...) wieder «weich» werden.

Bildnachweis

Grafik erstellt von den Autoren 